\title{
Bar, stripe and spot development in sand-dwelling cichlids from Lake Malawi
}

\author{
Laura A. Hendrick', Grace A. Carter ${ }^{1}$, Erin H. Hilbrands', Brian P. Heubel ${ }^{1}$, Thomas F. Schilling² \\ and Pierre Le Pabic ${ }^{1 *}$ (1)
}

\begin{abstract}
Background: Melanic patterns such as horizontal stripes, vertical bars and spots are common among teleost fishes and often serve roles in camouflage or mimicry. Extensive research in the zebrafish model has shown that the development of horizontal stripes depends on complex cellular interactions between melanophores, xanthophores and iridophores. Little is known about the development of horizontal stripes in other teleosts, and even less is known about bar or spot development. Here, we compare chromatophore composition and development of stripes, bars and spots in two cichlid species of sand-dwellers from Lake Malawi-Copadichromis azureus and Dimidiochromis compressiceps.
\end{abstract}

Results: (1) In D. compressiceps, stripes are made of dense melanophores underlaid by xanthophores and overlaid by iridophores. Melanophores and xanthophores are either loose or absent in interstripes, and iridophores are dense. In C. azureus, spots and bars are composed of a chromatophore arrangement similar to that of stripes but are separated by interbars where density of melanophores and xanthophores is only slightly lower than in stripes and iridophore density appears slightly greater. (2) Stripe, bar and spot chromatophores appear in the skin at metamorphosis. Stripe melanophores directly differentiate along horizontal myosepta into the adult pattern. In contrast, bar number and position are dynamic throughout development. As body length increases, new bars appear between old ones or by splitting of old ones through new melanophore appearance, not migration. Xanthophore and iridophore distributions follow melanophore patterns. (3) Metamorphic pigmentation arises in cichlids in a fashion similar to that described in zebrafish: melanophore progenitors derived from the medial route of neural crest migration migrate from the vicinity of the neural tube to the skin during metamorphosis.

Conclusion: The three pigment cell types forming stripes, bars and spots arise in the skin at metamorphosis. Stripes develop by differentiation of melanophores along horizontal myosepta, while bars do not develop along patent anatomical boundaries and increase in number in relation with body size. We propose that metamorphic melanophore differentiation and migratory arrest upon arrival to the skin lead to stripe formation, while bar formation must be supported by extensive migration of undifferentiated melanophores in the skin.

Keywords: Cichlid, Pigment pattern, Melanophore, Xanthophore, Iridophore, Evolution, Pigmentation development, Neural crest, Morphogenesis

\footnotetext{
*Correspondence: lepabicp@uncw.edu

${ }^{1}$ Department of Biology and Marine Biology, University of North Carolina

Wilmington, 5216 Randall Drive, Wilmington, NC 28403, USA

Full list of author information is available at the end of the article
} 


\section{Background}

The evolutionary diversification of body coloration is a fascinating subject because body color is at the center of multiple selective pressures, such as light protection, female mate choice and predation. As such, coloration likely plays an important role in the creation and maintenance of biodiversity, yet the genetic and developmental changes required for the evolution of new patterns with adaptive value remain largely unknown.

Various mechanisms underlie the diversity of colors and color patterns observed among vertebrates. The melanocyte is the only pigment cell type, or chromatophore, found in birds and mammals [1]. Melanocytes may produce different kinds of melanin pigment to produce colors ranging from yellowish (pheomelanin) to black (eumelanin). Other colors seen in birds and mammals result from food-derived pigments interacting with skin extracellular matrix $[2,3]$. A greater diversity of chromatophore types underlies body coloration in reptiles, amphibians and fish, with seven types described in fish: xanthophores, erythrophores, iridophores, leucophores, melanophores, cyanophores and erythro-iridophores [4-7].

Vertebrate chromatophores are derived from an embryonic population of pluripotent and highly migratory cells called neural crest (NC) cells. These cells emerge from the dorsal neural tube toward the end of neurulation and migrate throughout the embryo to give rise to the skeletal and connective tissues of the face, neurons and glia of the peripheral nervous system and chromatophores, among many other derivatives [8]. Trunk NC cells migrate through two distinct routes previously thought to be associated with distinct fates. Chromatophore progenitors migrate along a "lateral" route between surface ectoderm and somite, while neuronal and glial progenitors migrate along a "medial" route between somite and neural tube [9-11]. Recent evidence has shown that chromatophores also arise from $\mathrm{NC}$ cells that travel along the medial route in zebrafish and give rise to melanophores and iridophores during metamorphosis or regeneration $[12,13]$. It is unknown if this mechanism is conserved in other teleosts or other vertebrate clades.

The highly diverse patterns of chordate body colors result from the particular distribution of pigments and/or chromatophores and in many instances of their particular combinations and overlapping patterns in the skin. The numerous studies focusing on zebrafish horizontal stripe formation have made the developmental and genetic basis of this complex pattern the best understood of all animals. Three chromatophore types, black melanophores, yellow xanthophores and silver/blue iridophores make up the zebrafish stripe pattern. Dark stripes are composed of all three chromatophore types with melanophores providing darkness, while iridophores provide iridescence and blue hue or green hue when combined with xanthophores. Light interstripes are made of xanthophores overlaid on iridophores [14, 15]. This adult pattern arises gradually during metamorphosis, spanning the period of 3 to $6 / 7$ weeks post-fertilization $[16,17]$. A key finding in zebrafish stripe development is that the distribution of each chromatophore depends on interactions with other chromatophore types. Melanophores prevent the spreading of dense iridophores into dark stripe territory, iridophores and xanthophores promote melanophore numbers, yet xanthophores also prevent spreading of iridophores into dark stripe regions, maintain the integrity of melanophore stripes and prevent spreading of melanophores in light stripe regions [1821]. It is thus crucial to consider all chromatophore types when attempting to understand body color development in other organisms. While horizontal stripes are commonly found in other teleost species, the development of other color pattern elements, such as vertical bars and spots, remains poorly studied. It is unknown if the cellcell interactions described in danios exist in other genera and if so, how they generate complex body coloration. In addition, only a handful of studies have identified the types of genetic and developmental changes driving the evolution of such complex patterns [22-24].

The great diversity of color patterns seen in the cichlid species flocks of East African rift lakes makes them great models to elucidate the developmental basis of previously unexplored patterns. The young ages of the species assemblies found in lakes Victoria (500,000 years) and Malawi (4 million years; [25]) make them amenable to genetic mapping by allowing the production of fertile hybrids in laboratory conditions. Moreover, the presence of highly similar yet independently evolved color patterns in species from these lakes offers great opportunities to elucidate the mechanisms underlying convergent evolution [26] as recently demonstrated by Kratochwill et al. [23].

Here, we describe and compare the development of the distinct color patterns of two cichlid species from Lake Malawi, Copadichromis azureus and Dimidiochromis compressiceps, in order to identify the developmental differences underlying their divergent color patterns. The color pattern of $C$. azureus includes three dark spots and a series of vertical dark bars. The $D$. compressiceps pattern includes three horizontal dark stripes. Similar to zebrafish, we find that three pigment cell types underlie the color patterns of both species, melanophores, xanthophores and iridophores, with different distributions in D. compressiceps and C. azureus adults. The time of first appearance of each chromatophore type is similar in $D$. compressiceps and C. azureus. Development of the adult 
pattern begins during larval development, essentially defining the onset of metamorphosis. Xanthophores appear first in both species, followed by melanophores, and finally iridophores. D. compressiceps stripes lay directly on top of horizontal myotome boundaries, while C. azureus vertical stripes do not clearly correlate with patent anatomical boundaries. We demonstrate by in situ hybridization that migration of NC cells takes place between $42 \mathrm{~h}$ post-fertilization (hpf) and 54hpf in both species along the lateral and medial routes and that, similar to zebrafish, pigment progenitors migrate from the vicinity of the neural tube to the skin during metamorphosis.

\section{Results}

\section{Adult pigmentation in C. azureus and $D$. compressiceps}

The $C$. azureus body pigment pattern consists of the superimposition of two patterns commonly found in Lake Malawi, but also lakes Victoria and Tanganyika [26-28]: (1) an alternating series of dark bars and light interbars, and (2) three black spots (S1-3; Fig. 1a). Young adults have nine bars (b1-9) and eight interbars (i1-8; Fig. 1a), while more bars are observed on larger individuals. A series of five melanic patches variably aligned with vertical bars are present at the base of the dorsal fin. Individuals can modify the contrast between bars and interbars by changing bar darkness. A silver sheen covers the entire body with the exception of the spots, suggesting the widespread presence of iridophores. This pattern is seen in both males and females, although the silver sheen becomes metallic blue when males become dominant. In order to identify (1) what types of chromatophores are present in C. azureus and (2) how their particular arrangement creates the $C$. azureus pattern, we observed the skin of live individuals at various optical magnifications. Because pigment cells overlap one another in the skin, live specimens were photographed before and after $\mathrm{L}$-adrenaline treatment to induce melanin granule aggregation in order to obtain a clear relationship between the abundance of particular chromatophores and the larger patterns observed on adult specimens: bars, interbars and spots. Three pigment cell types were identified: melanophores (dark brown/black), xanthophores (yelloworange) and iridophores (silvery-white; Fig. 1b-g). All three chromatophore types are found in both the dark bars and the light interbars (Fig. 1d, e), as well as the spots (Fig. 1f, g). Melanophores were 23\% more abundant in bars $\left(99.4 \mathrm{cell} / \mathrm{mm}^{2}\right)$ than interbars $\left(81 \mathrm{cell} / \mathrm{mm}^{2}\right.$; Fig. 1h), and cell size was larger in bars (Fig. 1d). Xanthophore abundance was $11 \%$ greater in bars $\left(61.3 \mathrm{cell} / \mathrm{mm}^{2}\right)$ than interbars $\left(55.3 \mathrm{cell} / \mathrm{mm}^{2}\right.$; Fig. 1d-e, h). Iridophores were generally overlaid on melanophores in spots, bars and interbars (Fig. 1d, f), where they seemed to provide greater cover although they could not be counted. Melanophore and xanthophore abundance was similar in spots as in bars $\left(89.7 \mathrm{cell} / \mathrm{mm}^{2}\right.$ and $68.3 \mathrm{cell} / \mathrm{mm}^{2}$; Fig. $1 \mathrm{~g}$, h), although melanophores were darker in spots (Fig. 1i).

The $D$. compressiceps body pigment pattern is also found on numerous cichlids from lakes Malawi, Tanganyika and Victoria and includes three horizontal dark stripes separated by light silvery interstripes (Fig. 1j, k; $[27,28])$. An additional dark stripe runs along the dorsal midline (Fig. 1j, k). Dark stripes tend to disappear in dominant males as a metallic blue sheen develops over the entire body. As in C. azureus, melanophores, xanthophores and iridophores were identified by microscopy on live specimens before and after L-adrenaline treatment (Fig. 1k-o). All dark stripes contain the three chromatophores, while dorsal interstripes contain iridophores and melanophores, and ventral interstripes contain iridophores alone (Fig. 1k-o). Differences in melanophore density differentiate the stripes: melanophore density is the highest in the midline stripe (SML), intermediate in the dorsolateral (SDL) and dorsal (SD) stripes and lowest in the ventrolateral stripe (SVL), resulting in a discontinuous appearance (Fig. $1 j-$ p).

\section{Pre-metamorphic pigmentation development in $D$. compressiceps and C. azureus}

In order to understand how each species-specific pigment pattern develops, live specimens from both species were imaged daily throughout embryonic and larval

\footnotetext{
(See figure on next page.)

Fig. 1 Adult pigmentation in C. azureus and D. compressiceps. a Vertical bars b1-9, interbars i1-8 and spots S1-3 in C. azureus. b, c Higher magnification images of bars/interbars and spots in C. azureus before (b) and after (c) adrenaline treatment. $\mathbf{d}$, e Melanophores (black), xanthophores (orange) and iridophores (silver) in bars and interbars of C. azureus before (d) and after (e) adrenaline treatment. f, $\mathbf{g}$ Melanophores, xanthophores and iridophores of spot in C. azureus before $(\mathbf{f})$ and after $(\mathbf{g})$ adrenaline treatment. $\mathbf{h}$ Melanophore and xanthophore average densities in bars, interbars and spots $(n=3)$ in C. azureus. Error bars represent standard deviations. i Melanophore darkness in spot and bar expressed as integrated density. $\mathbf{j}$ Stripes and interstripes in D. compressiceps adult. $\mathbf{k}$, I Higher magnification images of stripes and interstripes in D. compressiceps flank before (k) and after $(\mathbf{I})$ adrenaline treatment. $\mathbf{m}$-o Melanophores, xanthophores and iridophores of stripes SDL and SML and interstripe X1DL before $(\mathbf{k}, \mathbf{m})$ and after $(\mathbf{I}, \mathbf{n})$ adrenaline treatment. $\mathbf{o}$ Lack of xanthophores in interstripe X1DL visualized under fluorescent light (488 nm). p Melanophore and xanthophore average densities in SDL, SML and SVL $(n=3)$ in D. compressiceps. Error bars represent standard deviations. $\mathbf{a}, \mathbf{j}$ Scale $=1 \mathrm{~cm} . \mathbf{b}, \mathbf{d}, \mathbf{k}$, I Scale $=1 \mathrm{~mm} \cdot \mathbf{d}-\mathbf{g}$ and $\mathbf{m}-\mathbf{o}$ Scale $=250 \mu \mathrm{m}$
} 

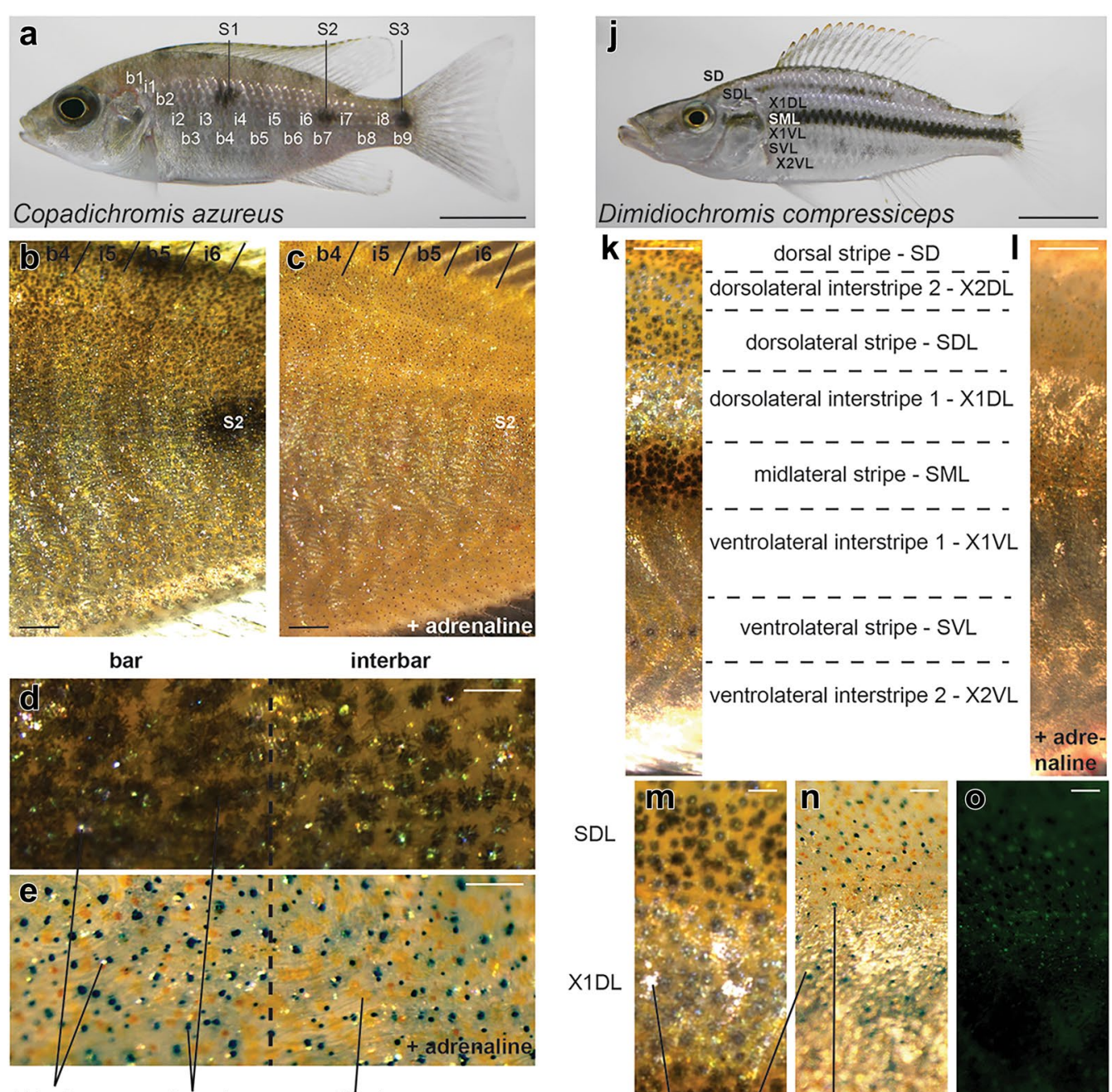

\section{Dimidiochromis compressiceps}

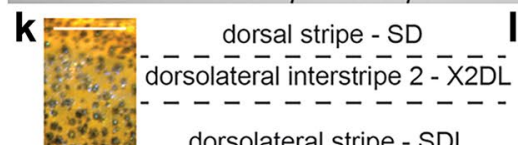

dorsolateral stripe - SDL

W. 1 dorsolateral interstripe $1-\mathrm{X} 1 \mathrm{DL}$

E. dorsolateral interstripe 1 - X1DL

midlateral stripe - SML
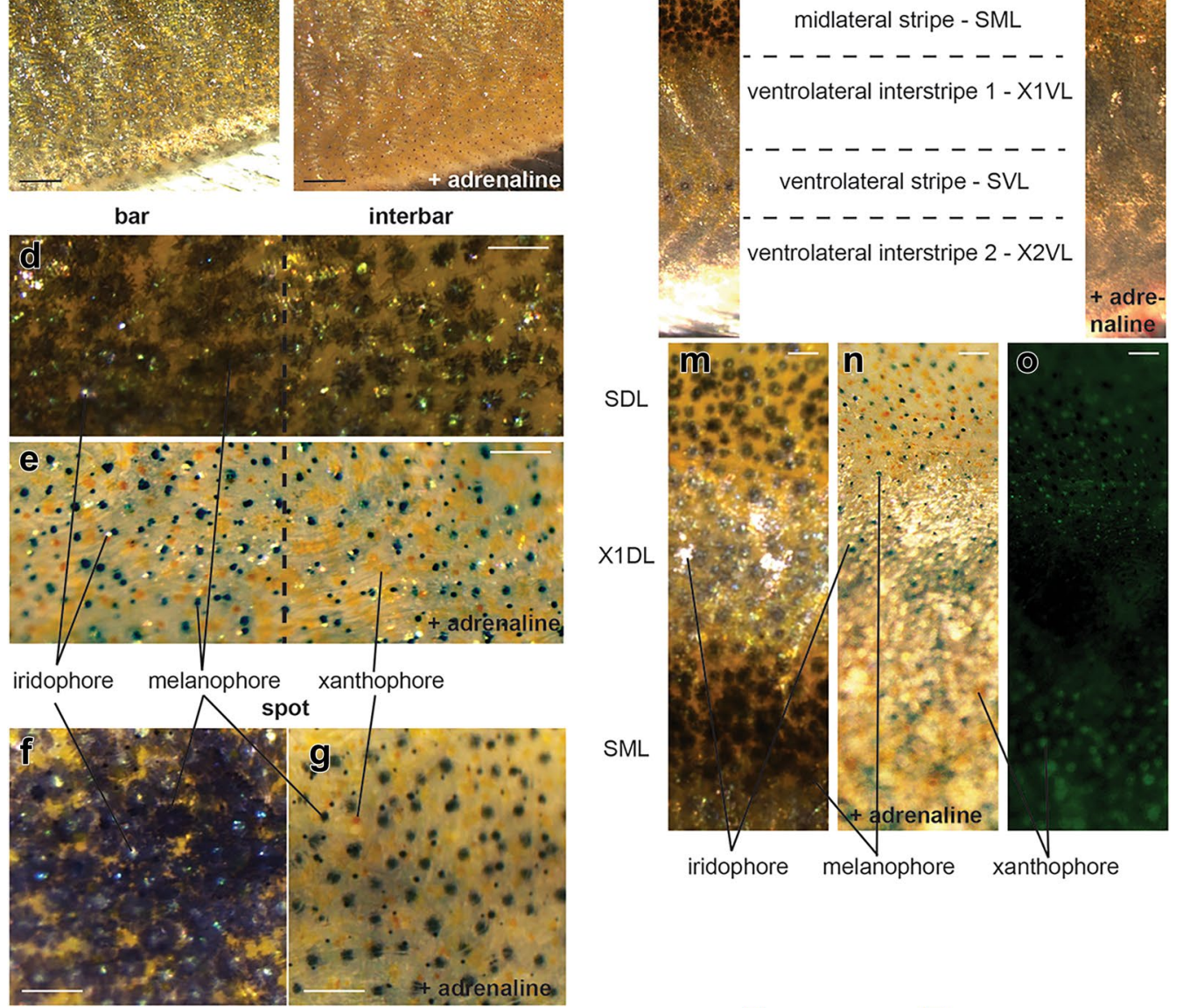

iridophore melanophore xanthophore

h $\square$ melanophore $\square$ xanthophore i
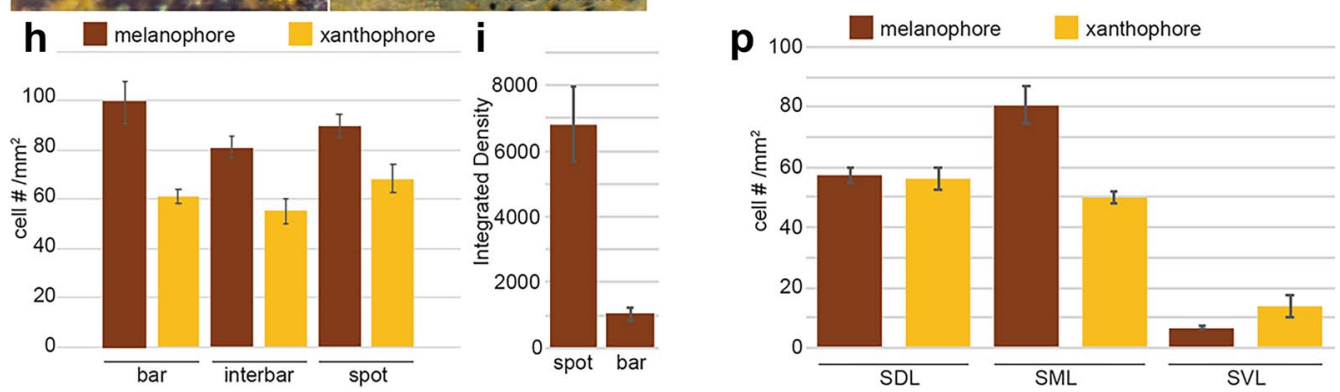
development, which ends after roughly 2 weeks of development when the yolk becomes fully depleted. Rates of development are extremely similar in $D$. compressiceps and C. azureus, and very similar to that described for the Nile tilapia [52]. Melanophores are the first pigment cells to differentiate in both species starting at $42 \mathrm{hpf}$ on the yolk (data not shown), followed by a 4-day period with minimal change to this pigmentation pattern (Fig. $2 \mathrm{a}-\mathrm{i}$, $\mathrm{m})$, except in the eye where epithelial melanization and iridophores were first observed at 4dpf (Fig. 2a, e) and 6dpf (Fig. 2c, g), respectively.

A new wave of skin pigmentation started at $6 \mathrm{dpf}$ (Fig. 2c, g), a day before overt morphological changes indicating the start of metamorphosis in both species such as separation of dorsal, caudal and anal fins and appearance of caudal fin ray elements (CFRE) (Fig. 3). Melanophores and xanthophores become visible on the dorsal head at $6 \mathrm{dpf}$ (Fig. 2c, g), followed by a progressive expansion of xanthophores alone along the dorsal trunk and tail at $7 \mathrm{dpf}$ (Fig. $4 \mathrm{a}-\mathrm{h}$ ). Xanthophore expansion proceeded ventrally over the next 3 days, reaching the lateral midline by $8 \mathrm{dpf} / 2$ CFRE (Fig. $4 \mathrm{e}-\mathrm{h}$ ) and the ventral midline by $10 \mathrm{dpf} / 5 \mathrm{CFRE}$ in both species (Fig. 4i-l). Following this full body coverage, xanthophore distribution became concentrated under bar melanophores in $C$. azureus (Fig. $4 \mathrm{~m}, \mathrm{n}$ ) or stripe melanophores in D. compressiceps (Fig. 4o, p) by the end of the larval stage (9CFRE).

Unlike xanthophores, metamorphic melanophores in the trunk (anterior to anus) and/or tail (posterior to anus) first appear deep in larvae of both species at $8 \mathrm{dpf} / 2 \mathrm{CFRE}$ - on the dorsal side of the neural tube (Fig. 2i, m). These deep melanophores appear along the entire length of the neural tube in C. azureus, while they are restricted to the tail region in $D$. compressiceps at this stage. Skin melanophores are first observed the next day (9dpf/3CFRE) in both species-at the base of the dorsal fin, and at different regions along the lateral midline (Figs. 2j, n and 5a, b).

\section{Metamorphic pigment pattern development in $C$. azureus}

Three distinct patches $(\mathrm{P})$ that prefigure the adult spots, but also the midparts of presumptive bars b4, b7 and b9 are observed in the skin at $9 \mathrm{dpf} / 3 \mathrm{CFRE}$ along the lateral midline of $C$. azureus: P1 on the trunk, P2 on the tail, and P3 on the caudal fin peduncle (Figs. 2j and 5a). New melanophore appearance within and around these three patches leads to increased melanophore density as well as progressive expansion of each patch until 12dpf/6CFRE (Figs. 2j-l, q and 5a, c). By 13dpf/7CFRE new melanophore appearance leads to the formation of 2 new patches: P4 between P1 and P2, and P5 between P2 and P3 (Figs. 2r and 5e). Interestingly, appearance of these new melanophore patches takes place between differentiating iridophore streaks (Figs. 2r, s and 5e). At $15 \mathrm{dpf} / 8 \mathrm{CFRE}$, a new melanophore patch, P6, appears anterior to P1 (Fig. 2s). The progressive definition of $C$. azureus bars and interbars occurs concomitantly with the appearance of a series of dark and light patches along the dorsal midline. Similar to lateral midline patches, the first pigment cells appearing at the dorsal midline are melanophores concentrated in three melanophore patches (D1-3) at the base of the dorsal fin at 11dpf/5CFRE (Figs. 21 and 5c). New melanophores increase the cell density around these three centers, and iridophores start appearing in the two regions separating the patches by 6CFRE/12dpf (Fig. 2q). By 13dpf/7CFRE, D1 becomes resegmented into D1a and D1b, and D2 into D2a and D2b by new iridophore patches (Figs. 2r and 5e). One more melanophore dorsal patch (D4) flanked by iridophores also appears on the caudal fin peduncle (Figs. $2 \mathrm{r}$ and $5 \mathrm{e})$. Some, but not all of these dorsal patches are dorso-ventrally aligned with the presumptive bars developing during the same period over C. azureus flanks.

In order to determine whether lateral patch melanophores migrate ventrally from dorsal patches as previously proposed in Mesoamerican cichlids [29] and more generally what cell behaviors underlie stripe morphogenesis, 4 C. azureus larvae were imaged daily from $10 \mathrm{dpf} / 4 \mathrm{CFRE}$ to $18 \mathrm{dpf} / 9 \mathrm{CFRE}$ at high magnification to follow individual melanophore behavior during bar formation (Fig. 6). Most melanophores did not move after appearing in the skin (Fig. 6a-i), and few mitoses were observed. Bars are thus shaped by the addition of new melanophores in C. azureus.

\section{Metamorphic pigment pattern development in $D$. compressiceps}

In contrast to $C$. azureus, the posterior half of the midlateral stripe in $D$. compressiceps first appears at 9dpf/3CFRE as a continuous stripe of melanophores along the lateral midline in the tail region (posterior to anus; Figs. 2n, 5b). Melanophores are also observed at the base of the dorsal fin at this stage, prefiguring the dorsal stripe (Fig. 5b). Melanophore density increases each day by the addition of new melanophores to posterior SML and SD (Figs. 2o, p, u-x and 5b, d, f, h). Melanophores forming the anterior half of SML appear dorsal to the yolk at 12dpf/6CFRE (Fig. 2u), along with those forming the dorsal (SD) and ventrolateral stripes (SVL). New melanophore additions through later stages increase cell density in each stripe without changing the pattern prefiguring the adult stripes (Fig. $2 \mathrm{u}-\mathrm{x}$ ).

Interstripe iridophores appear dorsal and ventral to the midlateral stripe at 13dpf/7CFRE (Figs. $2 \mathrm{v}$ and $5 \mathrm{f}$ ), and new iridophore additions through later stages give rise to 


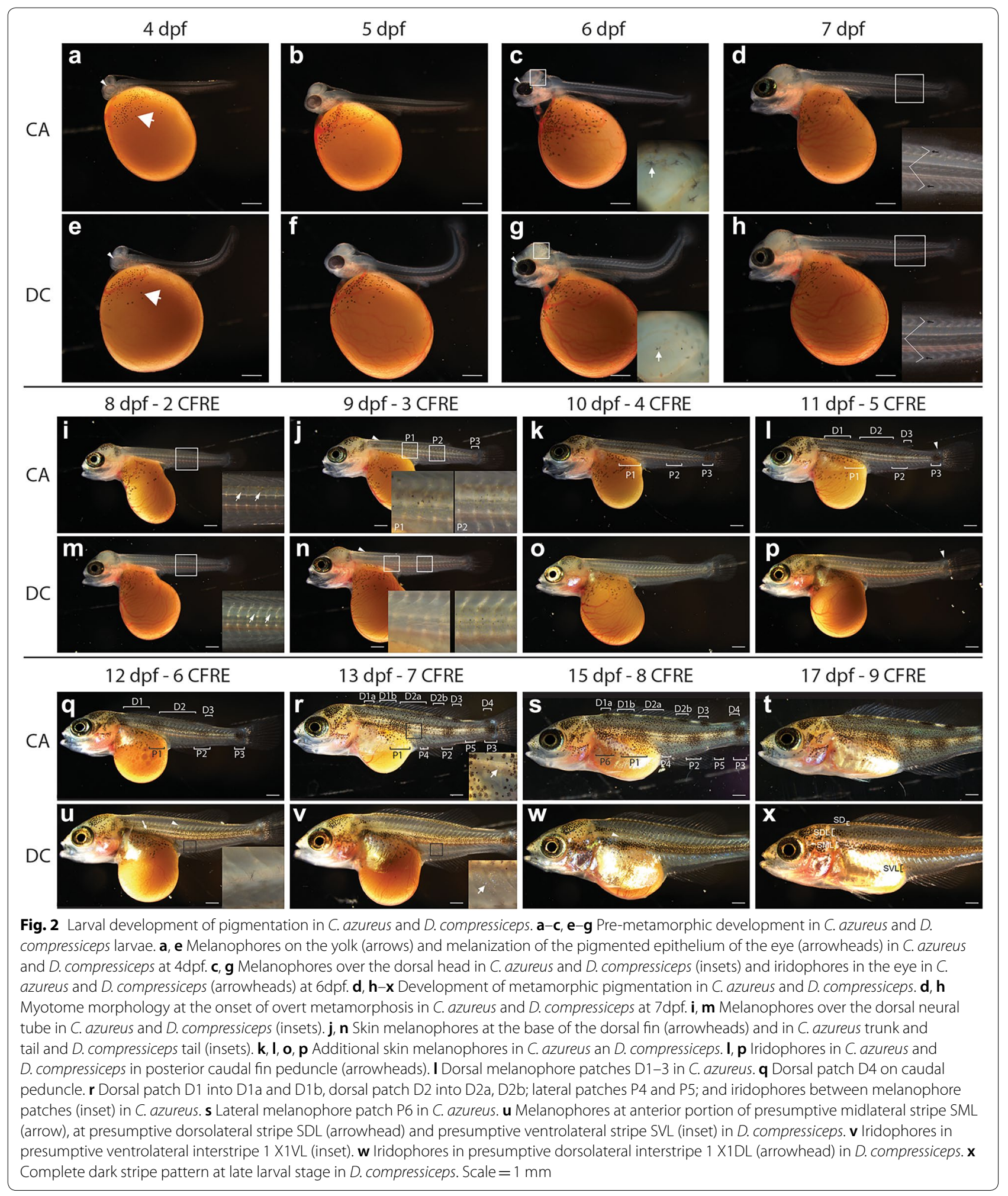



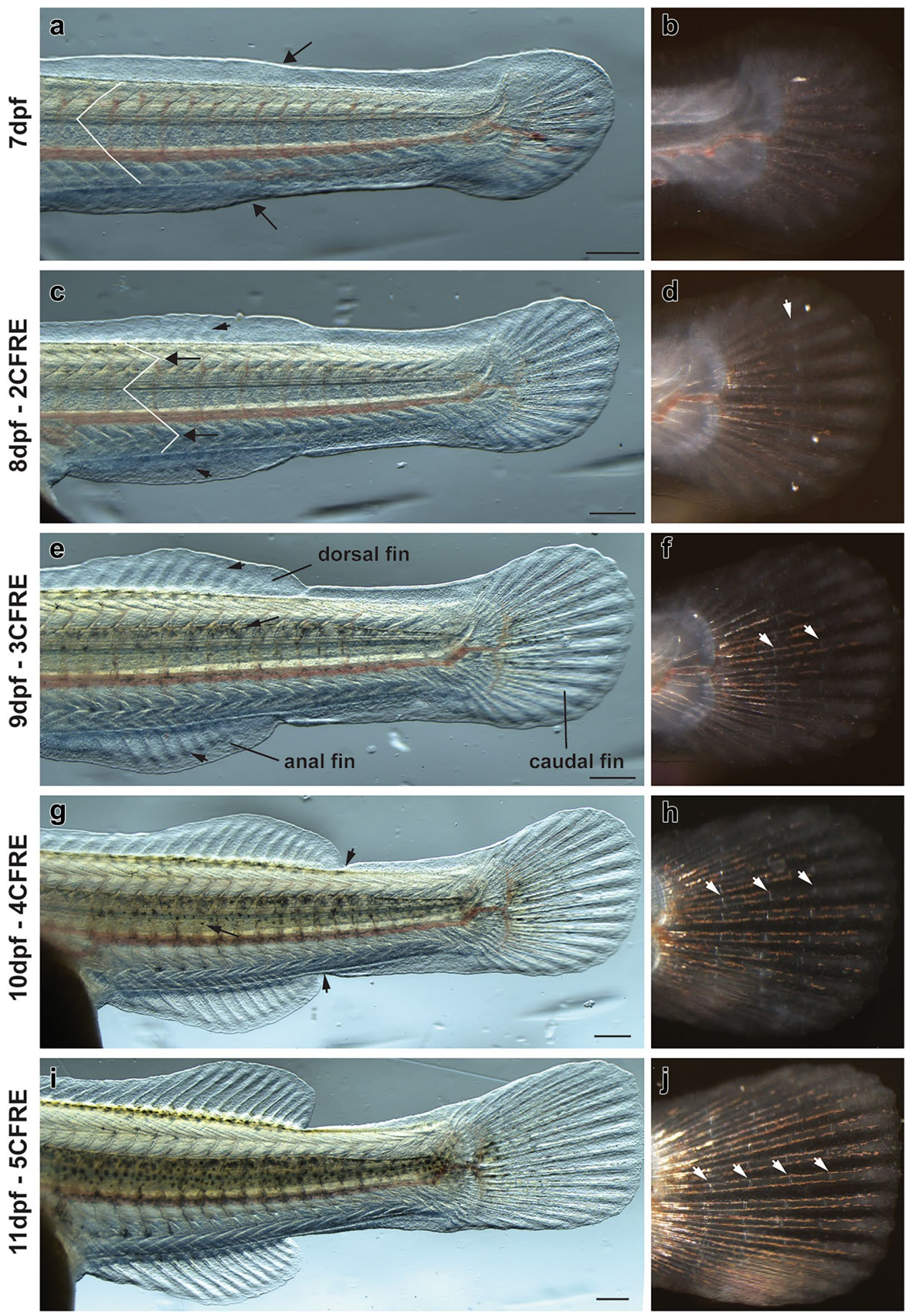

interstripes X1DL and X1VL (Figs. 2x and 5h). The adult D. compressiceps pigment pattern is essentially complete by the end of the larval period (17dpf/9CFRE) (Figs. $2 x$ and $5 \mathrm{~h})$.

\section{Timing of chromatophore progenitor development}

Based on the observed onset of adult pigmentation at metamorphosis in D. compressiceps and C. azureus, we next asked what are the embryonic origins of 


\section{(See figure on previous page.)}

Fig. 3 Morphological changes in median fins and myotomes during metamorphosis in D. compressiceps. a Bulges (arrows) in embryonic median fin prefigure the posterior ends of the dorsal and anal fin, respectively. "V" shape of myotomes indicated in white. b Unsegmented caudal fin ray elements. c Dorsal- and anal-fin ray condensations indicated by short arrows. Chevron shape of myotomes indicated by long arrows. $\mathbf{d} 2$ caudal fin ray elements separated by one joint (white arrow). e Fin ray elements lengthen in dorsal fin and anal fin (short arrows). Melanophores appear on dorsal neural tube (long arrow). $\mathbf{f}$ caudal fin ray elements separated by two joints (white arrows). $\mathbf{g}$ Dorsal and anal fins separated from caudal fin (short arrows). Melanophores appear in flank skin. $\mathbf{h} 4$ caudal fin ray elements separated by three joints (white arrows). i Progressive disappearance of larval fin tissue from dorsal and ventral caudal fin peduncle. $\mathbf{j} 5$ caudal fin ray elements separated by four joints (white arrows). The timing of metamorphosis is the same in C. azureus as in D. compressiceps. Scale $=500 \mu \mathrm{m}$

metamorphic chromatophores in these cichlids. In zebrafish, metamorphic melanophore and iridophore progenitors arise from stem cell pools associated with dorsal root ganglia, which are derived from ventral/ medial route $\mathrm{NC}$ cells and replace embryonic/larval pigment cells $[12,13]$. These progenitors migrate to the skin along sensory axons traveling between myotomes [13]. In contrast, larval xanthophores are derived from dorsal/lateral route $\mathrm{NC}$ cells and remain in the skin at metamorphosis and into adulthood [17].

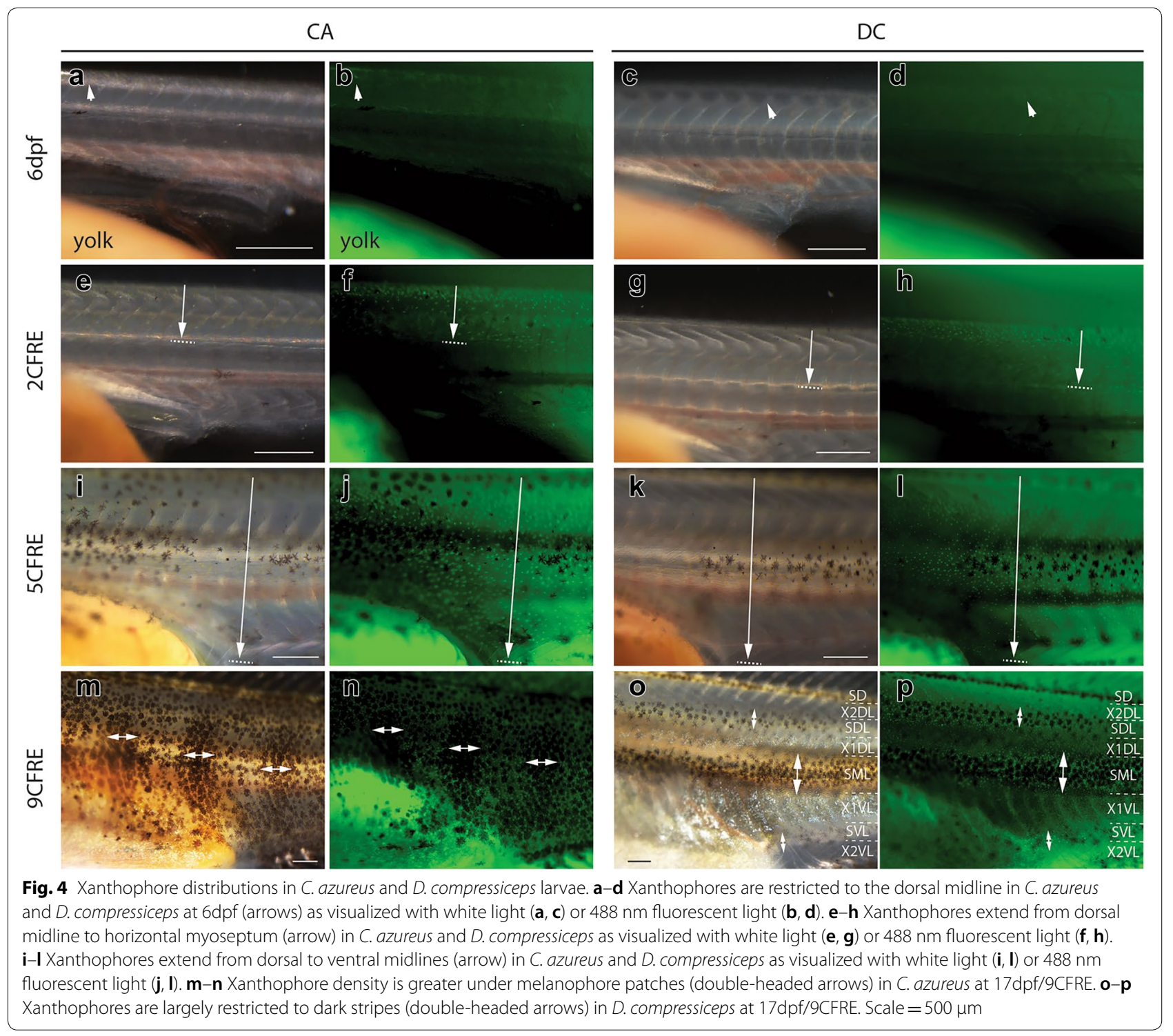



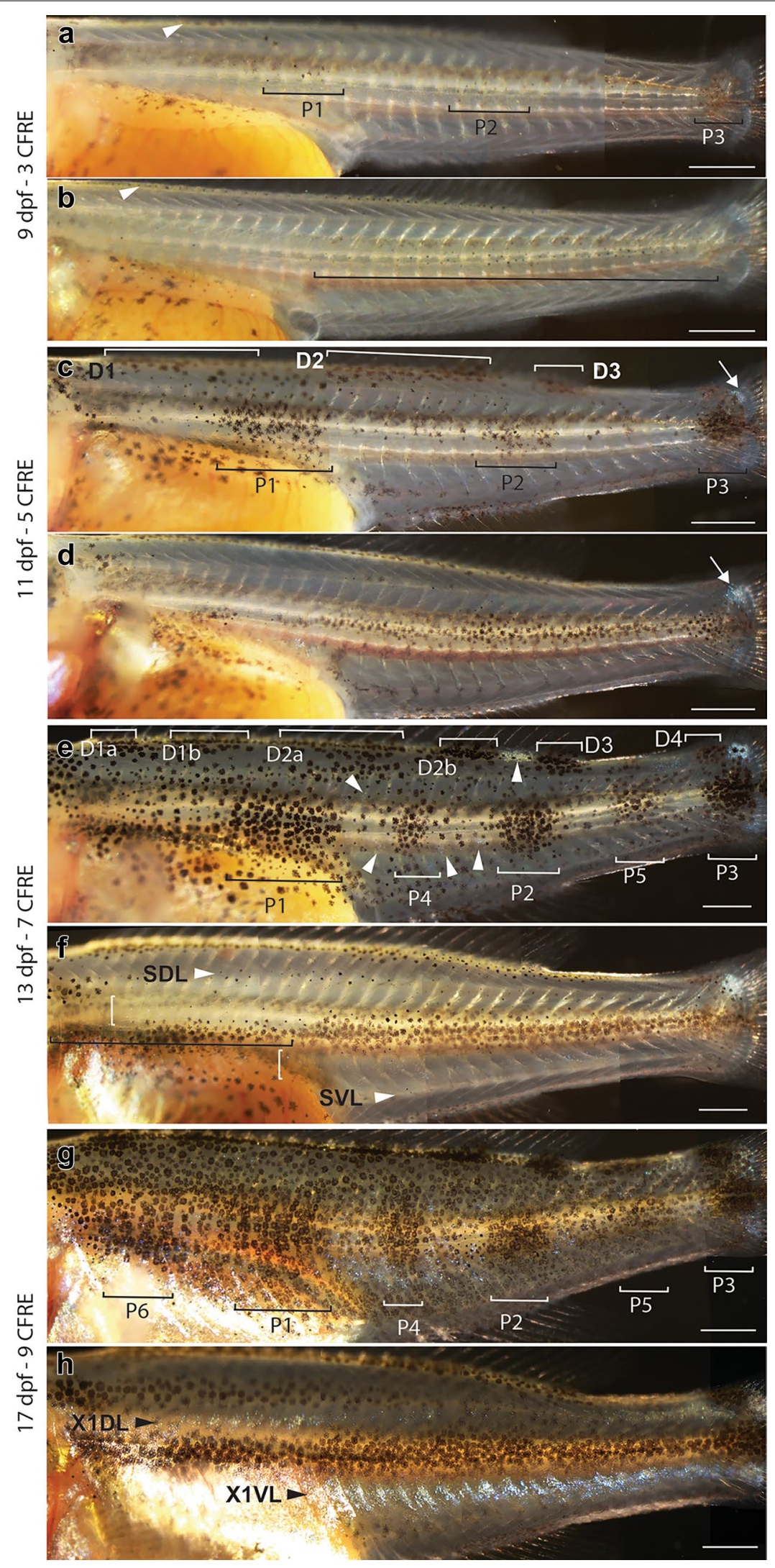
(See figure on previous page.)

Fig. 5 Melanophore and iridophore patterns in C. azureus and D. compressiceps larvae. a Melanophores in the flank in C. azureus-patches P1-3 and base of dorsal fin (arrow). b Melanophores along tail lateral midline and base of dorsal fin (arrow) in D. compressiceps. c Melanophore additions to and around P1-3 in C. azureus; Resolution of dorsal midline melanophore band into D1, D2 and D3. Appearance of iridophore patch on dorsal caudal fin peduncle (arrow). d Melanophore additions to tail-portion of dark stripe S0 in D. compressiceps. Iridophore patch on dorsal caudal fin peduncle (arrow). e Resolution of dorsal melanophore patches D1 into D1a and D1b, and of D2 into D2a, D2b; appearance of D4 on dorsal caudal fin peduncle. Appearance of lateral patches P4 and P5. Iridophore patches between dorsal and lateral patches (arrowheads). f Melanophores along trunk portion of SML in D. compressiceps (black bracket). Melanophores at presumptive SDL and SVL (arrowheads). Iridophores dorsal and ventral to horizontal myoseptum (white brackets). $\mathbf{g}$ New melanophores around populated areas. Appearance of lateral melanophore patch P6 in C. azureus. $\mathbf{h}$ Iridophores become abundant at interstripes X1DL and X1VL in D. compressiceps. Scale $=1 \mathrm{~mm}$

In order to determine when chromatophore progenitors arise and migrate in D. compressiceps and C. azureus, we conducted in situ hybridization to detect the expression of NC- and chromatophore-specific markers at various developmental stages in $D$. compressiceps and $C$. azureus embryos. FoxD3 and sox 10 label premigratory and migratory NC cells, respectively [30-33]. Kit-l, mitfa, tyr and tyr-p label melanophore progenitors [34-36]; csf1ra, gch and $x d h$ label xanthophore progenitors $[18,37$, 38]; pnp4 and bmp4 label iridophore progenitors [39]. In addition, ednrb1a labels all three chromatophore types in zebrafish [40], as well as migratory NC cells in mammals [41].

Gene expression patterns were first examined at 36, $42,48,54$ and $60 \mathrm{hpf}$ based on previously described timing of cranial NC migration in another cichlid, the Nile Tilapia [42]. Sox 10 and foxD3 expression was detected at 36 and $42 \mathrm{hpf}$ in C. azureus (Fig. 7a-d) and D. compressiceps (data not shown). At $36 \mathrm{hpf}$, foxD3 is expressed in the hindbrain and in premigratory cranial NC cells, as well as two streams of NC cells just beginning to migrate bilaterally, which we interpret as the mandibular NC stream (directly posterior to the eye) and the post-otic NC stream (posterior to otic vesicle) (Fig. 7a). Sox10 expression was also detected in the hindbrain and cranial NC streams, albeit at lower intensity (Fig. 7b).

At $42 \mathrm{hpf}$, foxd3 expression was still detected in all three cranial NC streams, as well as in two distinct cell populations in the anterior trunk. One population was found ventrally between the neural tube and the first two somites, which we interpret as NC cells migrating along the medial/ventral route. A second population consisted of individual cells on top of the neural tube, which we interpret as $\mathrm{NC}$ cells migrating along the lateral route (Fig. 7c). At this and later stages, laterally migrating NC cells were present further posteriorly than medial/ ventrally migrating $\mathrm{NC}$ cells, consistent with an earlier onset of lateral migration during the anterior-posterior sequence of trunk NC migration, as described in other vertebrates, including zebrafish [43]. foxD3 was also detected in the medial parts of somites $3-7$, as previously described in zebrafish [44]. Sox10 expression at these stages was restricted to the pre- and post-otic streams of cranial NC but was also detected in medially migrating $\mathrm{NC}$ at the levels of somites $1-2$, and in laterally migrating $\mathrm{NC}$ cells further posteriorly, similar to foxd3 (Fig. 7d).

Trunk expression of foxD3 and sox10 at subsequent stages $(48,54 \mathrm{hpf})$ was detected in successively more posterior streams of NC cells. A notable difference between the medial and laterally migrating populations was the duration of foxd3 and sox 10 expression: expression of both was maintained in NC streams migrating medially but downregulated in streams migrating laterally (Fig. 7e, $\mathrm{f}, \mathrm{h}-\mathrm{k})$. Ednrb1a expression was also detected at 48 and $54 \mathrm{hpf}$ in both species. In the trunk, ednrb1a expression labeled medial NC streams similar to foxd3 and sox 10 (Fig. $7 \mathrm{~g}, 1, \mathrm{~m}$ ), and also lateral $\mathrm{NC}$ streams at $54 \mathrm{hpf}$ (Fig. 7l). Expression of foxd3, sox10 or ednrbla decreased by $60 \mathrm{hpf}$ and was no longer detected at 66 or $72 \mathrm{hpf}$, and none of the other markers listed above produced detectable signals from 36 to $72 \mathrm{hpf}$.

The undifferentiated progenitors of zebrafish metamorphic melanophores and iridophores reside at dorsal root ganglia until metamorphosis, when they migrate along nerve axons and between myotomes to the skin [13]. In order to determine whether similar pigment progenitors exist in cichlids, we conducted in situ hybridization on cryosections at premetamorphosis (96hpf) and metamorphosis stages (11dpf/5CFRE) using ednrbla as a pan-chromatophore marker and mitfa as a melanophore marker. Expression of both genes was first detected at 11dpf/5CFRE (Fig. 7n, o) along the neural tube, between myotomes, and in the skin, consistent with a migration of chromatophore progenitors from the vicinity of the neural tube to the skin during metamorphosis. No difference in mitfa or ednrb1a expression was detected between $D$. compressiceps and C. azureus at these stages.

\section{Discussion \\ Chromatophore distribution relative to anatomical landmarks}

How do spatial cues instruct pigment cells to differentiate in particular regions of the embryo or larva? Previous findings in zebrafish have shown that metamorphic 

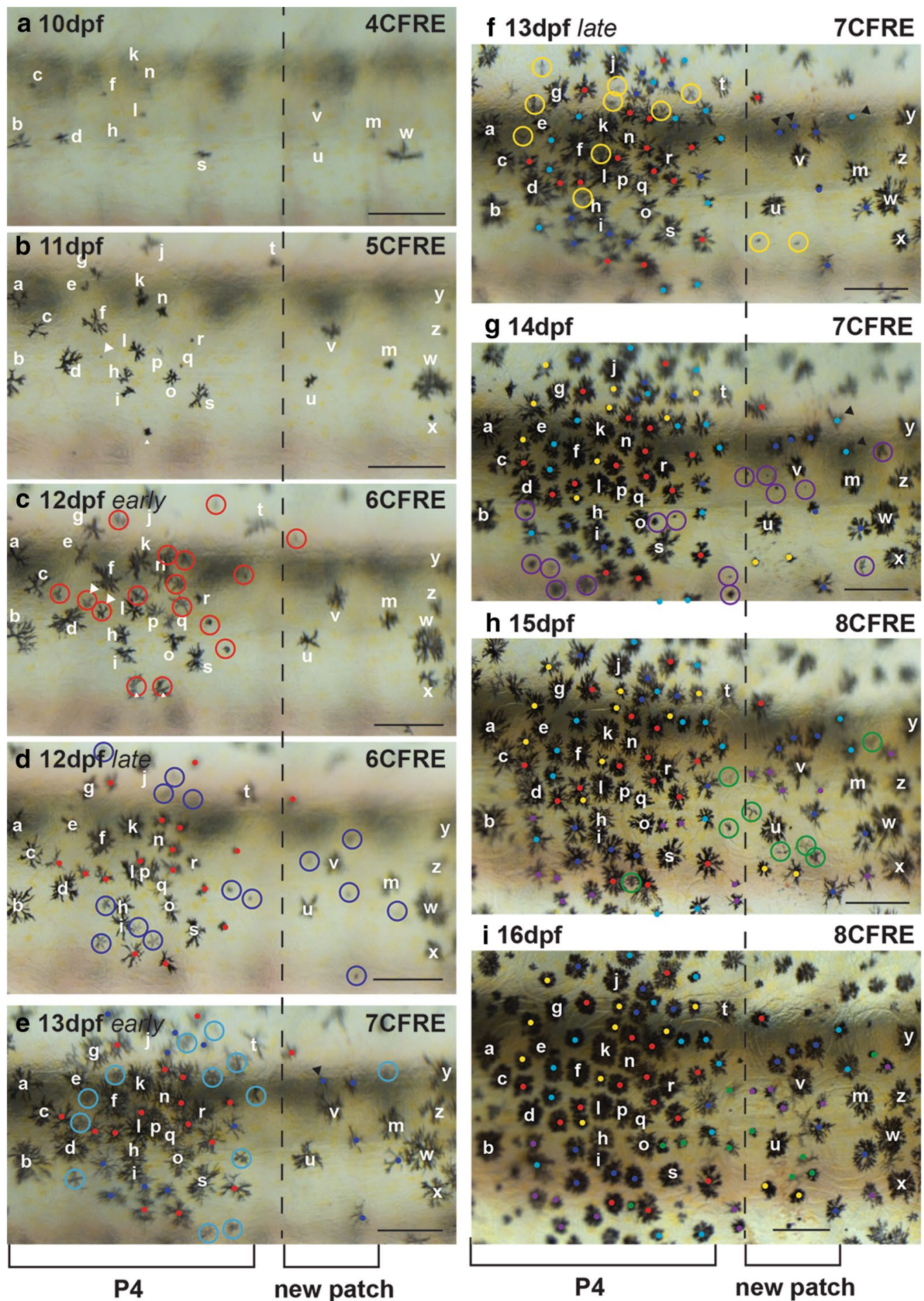

Fig. 6 Timelapse recording of bar melanophore development in C. azureus. a-i Melanophores of presumptive lateral patch P4 (left) and a posteriorly appearing patch over 6 days of development. Melanophores present at $10 \mathrm{dpf}(\mathbf{a})$ and $11 \mathrm{dpf}(\mathbf{b})$ are identified by the same letters at all timepoints. c New melanophores are circled in red at this stage (12 dpf early), and by red dots at subsequent stages. White arrowheads indicate daughter cells produced by melanophore mitosis. $\mathbf{d}$ New melanophores are circled in dark blue at this stage (12dpf late), and by dark blue dots at subsequent stages. e New melanophores are circled in light blue at this stage (13dpf early), and by light blue dots at subsequent stages. $\mathbf{f}$ New melanophores are circled in yellow at this stage (13dpf late), and by yellow dots at subsequent stages. Black arrowheads indicate daughter cells produced by melanophore mitosis. $\mathbf{g}$ New melanophores are circled in purple at this stage (14dpf), and by purple dots at subsequent stages. Black arrowheads indicate daughter cells produced by melanophore mitosis. $\mathbf{h}$ New melanophores are circled in green at this stage (15dpf), and by green dots at subsequent stages. $\mathbf{i}$ The relative positions of all melanophores examined remained largely unchanged from 10 to $16 \mathrm{dpf}$. Scale $=250 \mu \mathrm{m}$ 


\begin{abstract}
(See figure on next page.)
Fig. 7 Expression of neural crest (NC) cell- and pigment progenitor markers during embryonic development and later in metamorphosis in C. azureus. a-m Whole mount in situ hybridizations (ISH), anterior to the left, dorsal view (a-I) and lateral view (m). a, b foxd3 and sox 10 expression in presumptive hindbrain, mandibular- and post-otic NC streams at $36 \mathrm{hpf.} \mathbf{c}-\mathbf{f}, \mathbf{h}, \mathbf{i}$ foxd3 and sox 10 expression in trunk NC cells migrating through the lateral- and medial routes at 42 and $48 \mathrm{hpf}$. Foxd 3 is also expressed in somites. $\mathbf{g}$ ednrb 1 a expression in trunk NC cells migrating through the medial route at 48hpf. $\mathbf{h}-\mathbf{i}$ Higher magnifications of foxd3 and sox 10 expression in trunk NC cells migrating through the lateral-and medial routes at $48 \mathrm{hpf}$. $\mathbf{j}-\mathbf{k}$ foxd3 and sox 10 expression in trunk NC cells migrating through the lateral- and medial routes at 54hpf. Foxd3 expression is also detected in somites. I ednrbla expression in trunk NC cells migrating through the medial route at 54hpf. $\mathbf{m}$ Higher magnification of ednrbla expression in trunk NC cells migrating through the lateral route at 48hpf (somites 1-9 numbered). $\mathbf{n}-\mathbf{o}$ ISH on cryosections of 11dpf/5CFRE in C. azureus larvae. Ednrb1a and mitfa expression is detected at high levels in segmented structures located dorsal to the neural tube (\#), and at lower levels along myotome boundaries (arrowheads) and in the skin. e eye, $h b$ hindbrain, Ir lateral NC route, $m b$ myotome boundary, $m d$ mandibular NC stream, $m r$ medial NC route, my myotome, ov otic vesicle, post-otic post-otic NC stream, s somite. Scale $=250 \mu \mathrm{m}$
\end{abstract}

melanophore progenitors (melanoblasts) travel from dorsal root ganglia to the skin along axons between myotomes [13]. Here, we provide evidence for a similar mechanism in cichlids. This suggests a simple mechanism for horizontal stripe formation along horizontal myosepta: melanoblasts migrating to the skin along horizontal myosepta could give rise to horizontal stripes by differentiating in the skin upon arrival. In contrast, additional melanoblast migration would be required in the skin for vertical bar formation.

Dimidiochromis compressiceps dark stripes are clearly overlaid on horizontal myosepta: SML melanophores differentiate along the lateral midline myoseptum, stripe SD along the dorsal midline myoseptum, and stripes SDL and SVL along the dorsal and ventral lateral myosepta, respectively (Fig. 8b, d, f, h). In contrast, C. azureus vertical bars or spots arise from a progressively greater number of lateral melanophore patches that progressively elongate dorso-ventrally from new melanophores (Fig. 8a, c, e, g), without clear relationships with underlying myosepta. A major developmental difference resulting in the different color patterns of $C$. azureus and $D$. compressiceps may thus be in the migratory behavior of melanoblasts in the skin (Fig. 8i). Accordingly, the D. compressiceps pattern may result from the differentiation of melanoblasts into melanophores with limited migratory potential once they reach the skin, while the C. azureus pattern would require the migration of melanoblasts in the skin before differentiation into melanophores (Fig. 8i), since differentiated melanophores are distributed throughout the skin regardless of myoseptum position.

\section{Developmental regulation of bar number in old-world and new-world cichlids}

Vertical bars similar to those found in C. azureus are found in many cichlid species from both Africa and the Americas. Bar ontogeny was described in 40 species of neotropical cichlids of the subfamily Cichlasomatinae with various numbers of bars at adults stages [29]. Interestingly, the authors found that 9 vertical bars (including
2 in the head) develop first in all species examined, followed by species-specific bar divisions or fusions. Similar to $C$. azureus, bars form progressively starting with an initial pattern of lateral and dorsal midline melanophore clusters in Cichlasomatinae cichlid larvae. Dorsal and/or ventral migration of melanophores away from the clusters was reported to lead to the formation of bars. Our observation of individual melanophores in the skin of individual $C$. azureus larvae through several days of development did not show such migration of differentiated melanophores. Instead, supported exit points of metamorphic melanoblasts at horizontal myosepta suggest dorsal and ventral migration of melanophores through the skin in the undifferentiated state to result in the vertical bar pattern of $C$. azureus.

The timing of metamorphosis was also recently experimentally shown to modulate the number of vertical bars in a Mesoamerican cichlid, the convict cichlid Amatitlania nigrofasciata [45]. Consistent with a perturbation of metamorphosis [46], it was found that the timing and level of thyroid signaling activity affect (1) the timing of larval to juvenile coloration, and [2] the number of vertical bars. Individuals under elevated thyroid hormone levels from embryo to adult stages start pigmentation metamorphosis earlier than untreated individuals and develop 7 bars instead of 9, while individuals with lower thyroid hormone levels start pigmentation metamorphosis later and end up with 12 bars.

How does the timing of metamorphosis affect the number of bars? A common developmental pattern reported in barred Mesoamerican cichlids and here in C. azureus is the resolution of a few broad melanophore patches into numerous thin bars from larval to adult stages. In C. azureus new bars appear in growing larvae by new melanophore appearance in the skin-not rearrangement of already present melanophores. New bars form between old ones that have become spaced apart through body growth. Body growth also correlates with bar splitting-it creates new space between bar melanophores, which is filled by new melanophores within daughter bars but remains vacant between daughter bars. These 


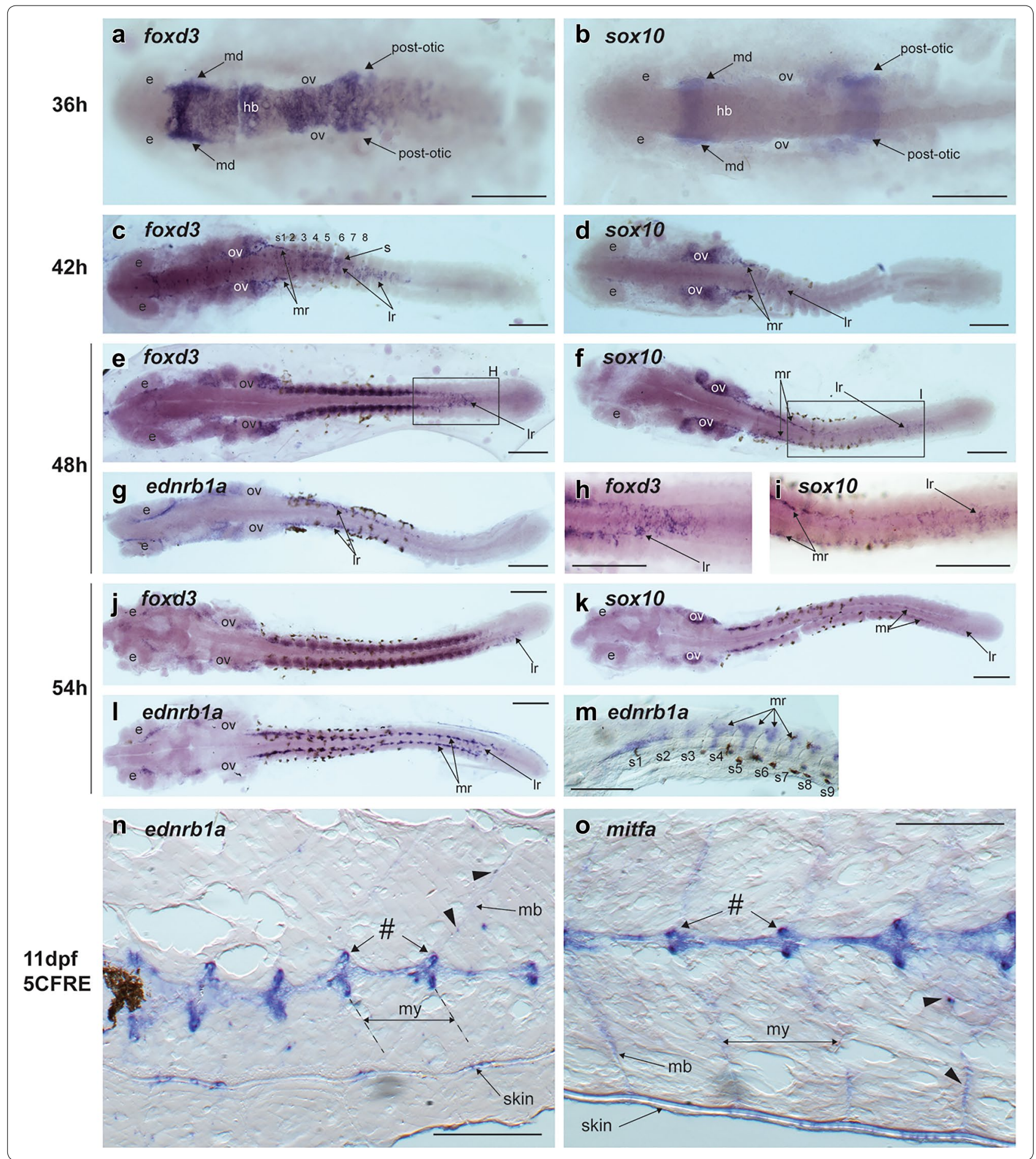

observations suggest that increase in physical space along the anterior-posterior axis is a positive regulator of bar number. The increase in bar number associated with delayed metamorphosis in the Mesoamerican cichlid $A$. nigrofasciata [45] also suggests that physical space at the time of first bar appearance is also a critical regulator of bar number. A. nigrofasciata individuals with delayed metamorphosis developed roughly the same number of bars as C. azureus at juvenile stages; however, these bars were thinner and thus more widely spaced, perhaps leaving more room for later-appearing bars. 


\begin{abstract}
(See figure on next page.)
Fig. 8 Summary of pigment pattern developmental differences in C. azureus and D. compressiceps. $\mathbf{a}$, $\mathbf{b}$ Xanthophores cover the body in both C. azureus and D. compressiceps at the onset of metamorphosis. $\mathbf{c}, \mathbf{d}$ Melanophores appear in the skin of C. azureus and D. compressiceps during metamorphosis, with a similar strip of metamorphic melanophores at the base of the dorsal fin in both C. azureus and D. compressiceps. c Patches of metamorphic melanophores appear on the flank skin in C. azureus and grow in size by new melanophore addition in and around each patch. d Metamorphic melanophores appear along the dorsolateral-, lateral midline- and ventrolateral myotome boundaries in D. compressiceps. e, $\mathbf{f}$ Chromatophore distribution at the end of the larval period in C. azureus and D. compressiceps. e Melanophore patches elongate dorso-ventrally and become resolved into bars flanked by iridophores in C. azureus. New bars appear by appearance of new melanophores. Xanthophore density increases under bar melanophores. $\mathbf{f}$ New melanophores appear largely along already formed horizontal stripes in $D$. compressiceps. Xanthophores increase in density under stripe melanophores and disappear from interstripe regions. Iridophores appear in interstripe regions. $\mathbf{g}, \mathbf{h}$ Pigment cell patterns in C. azureus and D. compressiceps adults. $\mathbf{g}$ In C. azureus bars are composed of dense melanophores lying on dense xanthophores and under loose iridophores. Melanophore densities are lower in interbars than in bars, while iridophore density is greater. Xanthophore distribution is similar in bars and interbars. $\mathbf{h}$ In D. compressiceps stripes are composed of melanophores and xanthophores in varying densities (greatest in SML, intermediate in SDL, lowest in SVL). Ventral interstripes are composed of iridophores exclusively, while dorsal interstripes also contain loose melanophores. i Model of differential migration of metamorphic melanoblasts in the skin of C. azureus (left) versus D. compressiceps (right). We propose that migratory potential is a major developmental difference in metamorphic melanoblast behavior between C. azureus and $D$. compressiceps. Under this model, C. azureus metamorphic melanophores do migrate dorsally and ventrally once they reach the skin, while skin migration is minimal in D. compressiceps, which results in melanophore positioning over horizontal myotome boundaries and formation of horizontal stripes
\end{abstract}

\section{Genetic determinants of cichlid color patterns}

The ontogeny of zebrafish body coloration is by far the best understood of all vertebrates because of the numerous mutant screens that have led to the identification of genes required for melanophore, xanthophore and iridophore development, but also for the interactions among and between these cell types and with their environment $[47,48]$. However, this understanding is largely restricted to the development of horizontal stripes. The diversity of cichlid color patterns in closely related species and their amenability to genetic mapping makes them a great model system to explore the developmental and genetic underpinnings of pigment pattern evolution in vertebrates. Research efforts have so far been far greater to identify the genetic factors regulating cichlid pigmentation than their developmental basis. Although most naturally occurring trait variants are under the control of multiple genes, as illustrated in Albertson et al. [49], two instances of monogenic traits were discovered in East African cichlids. Cis-regulatory variants affecting pax7a expression were found to be associated with the development of the orange-blotch camouflage pattern in Mbuna cichlids of Lake Malawi [50, 51]. More recently, a cis-regulatory variant affecting agrp2 expression was shown to determine the presence/absence of the horizontal dark stripe found in lake Victoria cichlids Haplochromis sauvagei and Pundamilia nyererei [23]. These authors also showed that difference in agrp2 expression reliably predicted the presence/absence of dark stripe in cichlids from other East African Lakes, thus demonstrating a clear case of convergence under the control of a single gene [23].

Here, we identified potential developmental differences underlying pigment pattern divergence in D. compressiceps and C. azureus. Identification of the loci regulating these developmental differences will constitute the next step toward a broader understanding of the developmental evolution of body coloration in vertebrates.

\section{Conclusion}

The three same chromatophore types make up D. compressiceps and C. azureus body coloration: melanophores, iridophores and xanthophores. In D. compressiceps, stripes are made of dense melanophores underlaid by xanthophores and overlaid by iridophores. Melanophores and xanthophores are either loose or absent in interstripes, and iridophores are dense. In C. azureus, spots and bars are composed of a chromatophore arrangement similar to that of stripes but are separated by interbars where density of melanophores and xanthophores is only slightly lower than in stripes and iridophore density appears slightly greater.

Differences in pigmentation between C. azureus and D. compressiceps arise at metamorphosis when melanophores appear in the skin as arrangements prefiguring stripes, bars or spots. In D. compressiceps, stripe melanophores differentiate along horizontal myosepta and the adult pattern is essentially in place by the end of the larval period. In contrast, the number of bars developing in C. azureus larvae is smaller than in adults. Bars appear as a series of lateral melanophore patches that progressively elongate dorso-ventrally by new melanophore additions. New bars appear either between old ones, or through splitting of old ones. The place of new melanophore appearance, not migration, determines new bar formation. Increase in xanthophore density in developing bars and stripes follows that of melanophores. Iridophores appear in interstripes after stripe formation, while 

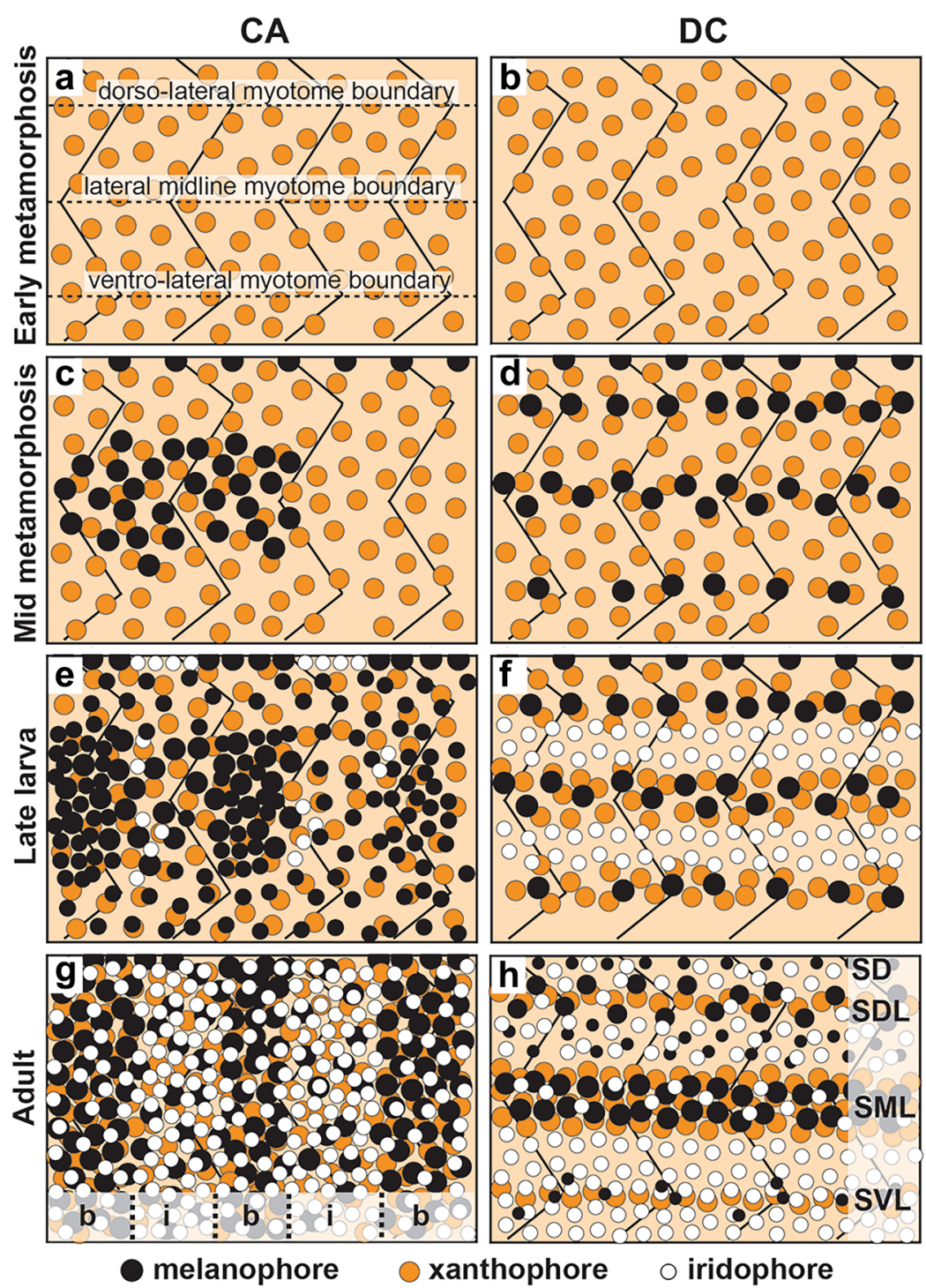

i Model-migration of metamorphic melanoblasts in skin

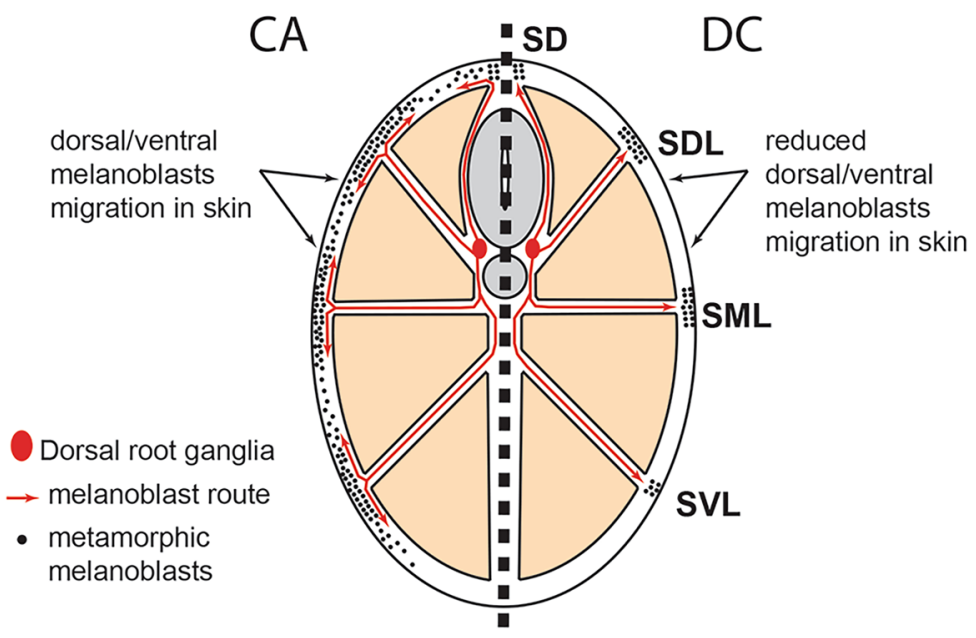


their appearance in interbars is concomitant with bar formation.

Analysis of neural crest migration by ISH indicates that melanophore progenitors migrate from the vicinity of the neural tube to the skin along myosepta at metamorphosis, as previously described in zebrafish [13]. Timelapse analysis of bar development during metamorphosis shows that melanophores do not migrate after differentiation. We thus propose that metamorphic melanophore differentiation and migratory arrest upon arrival to the skin lead to stripe formation, while bar formation must be supported by extensive dorsal and/or ventral migration of undifferentiated melanophores in the skin.

\section{Methods}

\section{Animal care}

All animals were reared and euthanized following protocols approved by the IACUC at the University of North Carolina, Wilmington. D. compressiceps and C. azureus brood stocks were purchased from a pet store and maintained separately at $28{ }^{\circ} \mathrm{C}$ in 55 - or 75 -gallon tanks. Adults were fed Northfin Food Cichlid Formula $3 \mathrm{~mm}$ sinking pellets. Fertilized eggs were collected from mouth-brooding females a few hours after fertilization. Embryos were then staged under a microscope, reared in cichlid egg tumblers and imaged daily until the end of the larval period (yolk depletion). Embryos and larvae were staged following Fujimura and Okada, 2007 [52].

\section{Image capture}

For imaging of live specimens, individuals were anesthetized in tank water using Tricaine (MP Biomedicals) following protocols approved by the IACUC at the University of North Carolina, Wilmington and immobilized on a drop of 3\% methyl cellulose (Alfa Aesar). L-Adrenaline treatment (Alfa Aesar) was carried out under the same conditions at a concentration of $4.5 \mathrm{mg} / \mathrm{mL}$. Chromatophore density averages reported in Fig. $1 \mathrm{~h}, \mathrm{p}$ were obtained from regions representative of each pattern in C. azureus $(n=3)$ or $D$. compressiceps $(n=3)$. To measure and compare melanophore darkness between bars and spots (Fig. 1i), images were [1] converted to greyscale and [2] inverted in Photoshop. Integrated density was then measured in Fiji, with darker regions displaying higher integrated density values than lighter regions. For timelapse imaging of $C$. azureus larvae, 4 specimens were imaged daily from $10 \mathrm{dpf} / 4 \mathrm{CFRE}$ to $18 \mathrm{dpf} / 9 \mathrm{CFRE}$ at high magnification to follow individual melanophore behavior during bar formation. Each specimen was anesthetized daily as described above, imaged and returned to its tank. Daily image capture was determined to be adequate to follow new melanophore appearance after multiple-daily image captures showed barely noticeable changes to the melanophore pattern. Melanophore migratory activity was assessed by recording [1] the position of melanophores relative to stable landmarks (myosepta) and [2] the relative positions of closest neighbors over the entire duration of the time-lapse imaging. The replacement of a single melanophore by 2 melanophores in successive time points was interpreted as a mitosis. All observations and images were made using a dissecting microscope Leica M165FC equipped with Planapo 1.6X M-series objective and Prior L200 fluorescent light source. Image capture was made with a Leica DFC7000T camera controlled by the LAS X software. Chromatophore counts were performed in FIJI. Image processing was performed in Adobe Photoshop.

\section{In situ hybridization}

Embryos and larvae were fixed and processed for whole mount in situ hybridization as described in Le Pabic

Table 1 Primers

\begin{tabular}{|c|c|c|c|}
\hline Gene & Forward primer & Reverse primer & $\begin{array}{l}\text { Amplicon } \\
\text { length (bp) }\end{array}$ \\
\hline foxd3 & TAAAGCCGCCTTACTCCTAC & GCGAGGAAGAGGAGGCAC & 654 \\
\hline sox 10 & CTGGGTCAGGAAGCGAGG & GCTCGAATGGGCGTAGTAT & 683 \\
\hline kit-l & TGTGTCCATTTCCTGCTGTT & TTCCTCCGTGATCTGACCTT & 647 \\
\hline mitfa & TCGCATTAAAGAGCTGGGAA & ACTGCAGTCATGTTTCACAG & 819 \\
\hline tyr & TGGACGCAACTCCCTTAATC & GCAATCAGAGCTCCCAGAAT & 708 \\
\hline$t y r-p$ & GTTTCCTGACCTGGCACA & CGGTCACAAACATCTCCAAG & 711 \\
\hline csfira & TCCCACCTTCAGCAAAATCT & TTCATCAGAGGCTGCTCTTC & 479 \\
\hline gch1 & CCAACCTACATGAACCAGCT & GCACACGTCTAATGTTGCTC & 861 \\
\hline$x d h$ & GGCAGCCTGTCATAACCATA & CACATCGGTCATCCAGTTCT & 716 \\
\hline pnp4 & GACGAATACCAGAAGACAGCT & CCTCGTAACTCTTCACCACC & 730 \\
\hline bmp4 & CACACTGCTGCATATGTTCG & CCGTAATTGGGAAGTCGTTC & 531 \\
\hline ednrbia & CCACAGAAATCCGAGACACT & GACAGATGCCATGTTGATGC & 854 \\
\hline
\end{tabular}


et al. [53]: embryos were fixed in 4\% PFA for 3 days at $4{ }^{\circ} \mathrm{C}$, rinsed in $\mathrm{PBT}$ and transferred to $100 \%$ methanol at $-20{ }^{\circ} \mathrm{C}$ for storage. For cryosections, embryos and larvae were processed as described in Albertson et al. [54]. In situ hybridization probes were generated from RTPCR amplified regions of C. azureus foxd3, sox10, kit-l, mitfa, tyr, tyr-p, csf1ra, gch, xdh, pnp4, bmp4, ednrb1a cDNAs using the primers listed in Table 1. mRNA was extracted from deyolked 54hpf C. azureus embryos. In situ hybridization was conducted using antisense probes prepared from pGem-t-easy vectors (Promega) containing cDNA fragments corresponding to $C$. azureus foxd3, sox10, kit-l, mitfa, tyr, tyr-p, csf1ra, gch, xdh, pnp4, bmp 4 and ednrb1a.

\section{Acknowledgements}

Not applicable.

\section{Authors' contributions}

LAH, GAC, EHH and BPH collected data, PLP designed the study, collected, analyzed and interpreted data and wrote the manuscript. TFS contributed in study design and writing the manuscript. All authors read and approved the final manuscript.

\section{Funding}

Research reported in this study was supported by NIH/NIDCR R01DE13828 to $\mathrm{PL}$ and TFS.

\section{Availability of data and materials}

The datasets used and/or analyzed during this study are available from the corresponding author on reasonable request.

\section{Ethics approval and consent to participate}

All animals were reared and euthanized following protocols approved by the IACUC at the University of North Carolina, Wilmington.

\section{Consent for publication}

Not applicable.

\section{Competing interests}

The authors declare that they have no competing interests.

\begin{abstract}
Author details
1 Department of Biology and Marine Biology, University of North Carolina Wilmington, 5216 Randall Drive, Wilmington, NC 28403, USA. ${ }^{2}$ Department of Developmental and Cell Biology, University of California, Irvine, 4109 Natural
\end{abstract} Sciences II, Irvine, CA 92697-2300, USA.

Received: 20 March 2019 Accepted: 27 July 2019

Published online: 12 August 2019

\section{References}

1. Caro T. The colours of extant mammals. Semin Cell Dev Biol. 2013;24:542-52

2. Roulin A, Ducrest AL. Genetics of colouration in birds. Semin Cell Dev Biol. 2013;24:594-608.

3. Kelsh RN, Harris ML, Colanesi S, Erickson CA. Stripes and belly-spots-a review of pigment cell morphogenesis in vertebrates. Semin Cell Dev Biol. 2009;20:90-104.

4. Olsson M, Stuart-Fox D, Ballen C. Genetics and evolution of colour patterns in reptiles. Semin Cell Dev Biol. 2013;24:529-41.

5. Grether GF, Kolluru GR, Nersissian K. Individual colour patches as multicomponent signals. Biol Rev. 2004;79:583-610.
6. Fujii R. The regulation of motile activity in fish chromatophores. Pigment Cell Res. 2000;13:300-19.

7. Goda M, et al. Integumental reddish-violet coloration owing to novel dichromatic chromatophores in the teleost fish, Pseudochromis diadema. Pigment Cell Melanoma Res. 2011;24:614-7.

8. Le Douarin NM, Kalcheim C. The neural crest. 2nd ed. Cambridge: Cambridge University Press; 1999.

9. Erickson CA, Weston JA. An SEM analysis of neural crest migration in the mouse. J Embryol Exp Morphol. 1983;74:97-118.

10. Raible DW, et al. Segregation and early dispersal of neural crest cells in the embryonic zebrafish. Dev Dyn. 1992;195:29-42.

11. Weston JA, Butler SL. Temporal factors affecting localization of neural crest cells in chicken embryo. Dev Biol. 1966;14:246.

12. Budi EH, Patterson LB, Parichy DM. Post-embryonic nerve-associated precursors to adult pigment cells: genetic requirements and dynamics of morphogenesis and differentiation. Plos Genet. 2011;7:e1002044.

13. Dooley CM, Mongera A, Walderich B, Nusslein-Volhard C. On the embryonic origin of adult melanophores: the role of ErbB and Kit signalling in establishing melanophore stem cells in zebrafish. Development. 2013;140:1003-13.

14. Hirata M, Nakamura K, Kanemaru T, Shibata Y, Kondo S. Pigment cell organization in the hypodermis of zebrafish. Dev Dyn. 2003;227:497-503.

15. Hirata M, Nakamura K, Kondo S. Pigment cell distributions in different tissues of the zebrafish, with special reference to the striped pigment pattern. Dev Dyn. 2005;234:293-300.

16. Singh AP, Schach U, Nusslein-Volhard C. Proliferation, dispersal and patterned aggregation of iridophores in the skin prefigure striped colouration of zebrafish. Nat Cell Biol. 2014;16:604.

17. Mahalwar P, Walderich B, Singh AP, Nusslein-Volhard C. Local reorganization of xanthophores fine-tunes and colors the striped pattern of zebrafish. Science. 2014:345:1362-4.

18. Maderspacher F, Nusslein-Volhard C. Formation of the adult pigment pattern in zebrafish requires leopard and obelix dependent cell interactions. Development. 2003;130:3447-57.

19. Frohnhofer HG, Krauss J, Maischein HM, Nusslein-Volhard C. Iridophores and their interactions with other chromatophores are required for stripe formation in zebrafish. Development. 2013;140:2997-3007.

20. Patterson LB, Parichy DM. Interactions with iridophores and the tissue environment required for patterning melanophores and xanthophores during zebrafish adult pigment stripe formation. Plos Genet. 2013;9:e1003561.

21. Nakamasu A, Takahashi G, Kanbe A, Kondo S. Interactions between zebrafish pigment cells responsible for the generation of Turing patterns. Proc Natl Acad Sci USA. 2009;106:8429-34.

22. Manceau M, Domingues VS, Mallarino R, Hoekstra HE. The developmental role of agouti in color pattern evolution. Science. 2011;331:1062-5.

23. Kratochwil CF, et al. Agouti-related peptide 2 facilitates convergent evolution of stripe patterns across cichlid fish radiations. Science. 2018;362:457.

24. Spiewak JE, et al. Evolution of endothelin signaling and diversification of adult pigment pattern in Danio fishes. Plos Genet. 2018;14:e1007538.

25. Delvaux D. Musée royal de I'Afrique Centrale (Tervuren), Département de Géologie et Minéralogie, rapport annuel, 1995, vol. 1993; 1994. p. 99-108.

26. Kocher TD, Conroy JA, McKaye KR, Stauffer JR. Similar morphologies of cichlid fish in lakes Tanganyika and Malawi are due to convergence. Mol Phylogenet Evol. 1993;2:158-65.

27. Barel CDN, van Oijen MJP, Witte F, Witte-Maas ELM. An introduction to the taxonomy and morphology of the haplochromine cichlids from Lake Victoria. Neth J Zool. 1977;27:381-9.

28. Seehausen O, Mayhew PJ, Van Alphen JJM. Evolution of colour patterns in East African cichlid fish. J Evol Biol. 1999;12:514-34.

29. Rican O, Musilova Z, Muska M, Novak J. Development of coloration patterns in neotropical cichlids (Teleostei: Cichlidae: Cichlasomatinae). Folia Zool. 2005;54:1-46.

30. Kelsh RN, Dutton K, Medlin J, Eisen JS. Expression of zebrafish fkd6 in neural crest-derived glia. Mech Dev. 2000;93:161-4.

31. Lister JA, et al. Zebrafish Foxd3 is required for development of a subset of neural crest derivatives. Dev Biol. 2006;290:92-104.

32. Stewart RA, et al. Zebrafish foxd3 is selectively required for neural crest specification, migration and survival. Dev Biol. 2006;292:174-88.

33. Dutton KA, et al. Zebrafish colourless encodes sox 10 and specifies nonectomesenchymal neural crest fates. Development. 2001;128:4113-25. 
34. Parichy DM, Rawls JF, Pratt SJ, Whitfield TT, Johnson SL. Zebrafish sparse corresponds to an orthologue of c-kit and is required for the morphogenesis of a subpopulation of melanocytes, but is not essential for hematopoiesis or primordial germ cell development. Development. 1999;126:3425-36.

35. Lister JA, Robertson CP, Lepage T, Johnson SL, Raible DW. Nacre encodes a zebrafish microphthalmia-related protein that regulates neural-crestderived pigment cell fate. Development. 1999;126:3757-67.

36. Johnson SL, Nguyen AN, Lister JA. Mitfa is required at multiple stages of melanocyte differentiation but not to establish the melanocyte stem cell. Dev Biol. 2011;350:405-13.

37. Parichy DM, Ransom DG, Paw B, Zon LI, Johnson SL. An orthologue of the kit-related gene fms is required for development of neural crest-derived xanthophores and a subpopulation of adult melanocytes in the zebrafish, Danio rerio. Development. 2000;127:3031-44.

38. Parichy DM, Turner JM. Temporal and cellular requirements for Fms signaling during zebrafish adult pigment pattern development. Development. 2003;130:817-33.

39. Lang MR, Patterson LB, Gordon TN, Johnson SL, Parichy DM. Basonuclin-2 requirements for zebrafish adult pigment pattern development and female fertility. Plos Genet. 2009;5:e1000744.

40. Parichy DM, et al. Mutational analysis of endothelin receptor b1 (rose) during neural crest and pigment pattern development in the zebrafish Danio rerio. Dev Biol. 2000;227:294-306.

41. Lee HO, Levorse JM, Shin MK. The endothelin receptor-B is required for the migration of neural crest-derived melanocyte and enteric neuron precursors. Dev Biol. 2003;259:162-75.

42. Le Pabic P, Stellwag EJ, Scemama J-L. Embryonic development and skeletogenesis of the pharyngeal jaw apparatus in the cichlid Nile tilapia (Oreochromis niloticus). Anat Rec. 2009;292:1780-800.

43. Raible DW, Eisen JS. Specification and commitment in zebrafish trunk neural crest development. Dev Biol. 1994;163:536.

44. Odenthal J, Nusslein-Volhard C. Fork head domain genes in zebrafish. Dev Genes Evol. 1998;208:245-58.
45. Prazdnikov DV, Shkil FN. Experimental evidence of the role of heterochrony in evolution of the Mesoamerican cichlids pigment patterns. Evol Dev. 2019;21:3-15.

46. McMenamin SK, Parichy DM. Metamorphosis in teleosts. Anim Metamorph. 2013;103:127-65.

47. Singh AP, Nusslein-Volhard C. Zebrafish stripes as a model for vertebrate colour pattern formation. Curr Biol. 2015;25:R81-92.

48. Parichy DM, Spiewak JE. Origins of adult pigmentation: diversity in pigment stem cell lineages and implications for pattern evolution. Pigment Cell Melanoma Res. 2015;28:31-50.

49. Albertson RC, et al. Genetic basis of continuous variation in the levels and modular inheritance of pigmentation in cichlid fishes. Mol Ecol. 2014;23:5135-50.

50. Roberts RB, Ser JR, Kocher TD. Sexual conflict resolved by invasion of a novel sex determiner in Lake Malawi cichlid fishes. Science. 2009;326:998-1001.

51. Roberts RB, Moore EC, Kocher TD. An allelic series at pax7a is associated with colour polymorphism diversity in Lake Malawi cichlid fish. Mol Ecol. 2017;26:2625-39

52. Fujimura K, Okada N. Development of the embryo, larva and early juvenile of Nile tilapia Oreochromis niloticus (Pisces: Cichlidae). Developmental staging system. Dev Growth Differ. 2007;49:301-24.

53. Le Pabic P, Stellwag EJ, Brothers SN, Scemama J-L. Comparative analysis of Hox paralog group 2 gene expression during Nile tilapia (Oreochromis niloticus) embryonic development. Dev Genes Evol. 2007;217:749-58.

54. Albertson RC, et al. Molecular pedomorphism underlies craniofacial skeletal evolution in Antarctic notothenioid fishes. BMC Evol Biol. 2010;10:4.

\section{Publisher's Note}

Springer Nature remains neutral with regard to jurisdictional claims in published maps and institutional affiliations.
Ready to submit your research? Choose BMC and benefit from:

- fast, convenient online submission

- thorough peer review by experienced researchers in your field

- rapid publication on acceptance

- support for research data, including large and complex data types

- gold Open Access which fosters wider collaboration and increased citations

- maximum visibility for your research: over $100 \mathrm{M}$ website views per year

At BMC, research is always in progress.

Learn more biomedcentral.com/submissions 